\title{
Needlescopy-assisted resection of pulmonary nodule after dual localisation
}

\author{
D-Y. Kang*, H.K. Kim*, Y.K. Kim\#", H.S. Yong ${ }^{\#}$, E-Y. Kang ${ }^{\#}$ and Y.H. Choi*
}

\begin{abstract}
The aim of this study was to evaluate the feasibility of dual localisation with hookwire and lipiodol before needlescopy-assisted resection for pulmonary nodule.

Computed tomography-guided dual marking was performed on 36 pulmonary nodules of 32 patients and needlescopy-assisted resection was performed monitored by C-arm fluoroscopy.

The mean age of the patients was $58 \pm 12$ (range 12-77) yrs. The mean size of the nodules was $7.5 \pm 3.7(3-17) \mathrm{mm}$. Their mean distance from the pleural surface was $7.3 \pm 7.5(0-35) \mathrm{mm}$. There were nine pure ground-glass opacity lesions, five semi-solid lesions and 22 solid lesions. The time of the dual localisation procedure was $13.1 \pm 4.8(7-23) \mathrm{min}$. Complications of the marking were pneumothorax in nine patients, and intrapulmonary bleeding in three. One hookwire dislodged during the operation. All nodules were successfully resected under needlescopy without conversion to a conventional thoracoscopy ( $5 \mathrm{~mm}$ or $10 \mathrm{~mm}$ thoracoscopy) or a minithoracotomy. There was no complication related to needlescopy-assisted resection.

Dual marking with hookwire and lipiodol is a safe and none time consuming procedure, and needlescopy-assisted lung resection for small nodules is technically feasible and useful for histological diagnosis and treatment.
\end{abstract}

\section{KEYWORDS: Diagnostics, general thoracic surgery, lung histology, open lung biopsy}

B ecause of the development of computed tomography (CT) and the more widespread use of chest CT in health screens, small peripheral pulmonary nodules which could not be detected by conventional radiography, or ground-glass opacity (GGO) lesions that require an exact diagnosis, are detected more frequently. However, because it is not easy to confidently differentiate a benign pulmonary lesion from a malignant one with CT criteria, a histopathological diagnosis is usually required. Performing a percutaneous or transbronchial biopsy for these lesions is often difficult when the lesion size is $\leqslant 15 \mathrm{~mm}$, when the lesions are GGO or when the lesions are located in a site that is difficult for these methods [1]. A surgical open lung biopsy has been considered the gold standard diagnostic modality, generally used as a final option due to its association with substantial morbidity and mortality, even with the advances in surgical technique and post-operative care. However, the frequency of thoracoscopic lung biopsies has increased because it is minimally invasive, results in fewer post-operative complications and reduces the duration of pleural drainage and the length of the hospital stay. Therefore, thoracoscopic surgical techniques have been used in diagnostic excisional biopsies, as well as in therapeutic resection. Moreover, as thoracoscopic lung biopsy techniques continue to improve, the size of the thoracoscopy has gradually decreased.

Recently, a lung biopsy using a needlescopy (2 mm thoracoscopy) and accompanying instruments has been introduced [2]. The needlescopic lung biopsy, which is much less invasive compared with other thoracoscopic procedures, may reduce post-operative pain, leave minimal scarring, and allow for more rapid recovery [3]. We have previously reported on the possibility of pulmonary nodule resection using a needlescopy [3].

However, successful resection using thoracoscopic biopsy, especially in the case of needlescopy, is difficult with small or deeply situated pulmonary nodules because of the lack of digital palpation. Furthermore, a bronchioloalveolar carcinoma with a GGO finding on CT frequently cannot be palpated or visualised, even in the case of lesions that are located just beneath the visceral pleura [4-8]. Therefore, pre-operative localisation of these pulmonary lesions is mandatory and various localisation techniques have been described, either pre-operatively (microcoils, dye, hookwire and lipiodol) [8-12] or intrathoracoscopically (finger palpation, intrathoracoscopic ultrasound and radio-guided surgery) $[13,14]$. However, to our knowledge, none of these methods have been widely adopted because of
AFFILIATIONS

Depts of *Thoracic and

Cardiovascular Surgery, and

"Radiology, Korea University Guro Hospital, Korea University College of Medicine, Seoul, Korea.

CORRESPONDENCE

H.K. Kim

Dept of Thoracic and Cardiovascular

Surgery, Korea University Guro

Hospital

Korea University College of Medicine

97 Guro-donggil

Guro-gu

Seoul 152-703

Korea

E-mail: kimhyunkoo@korea.ac.kr

Received:

Feb 082010

Accepted after revision:

April 292010

First published online:

June 072010 

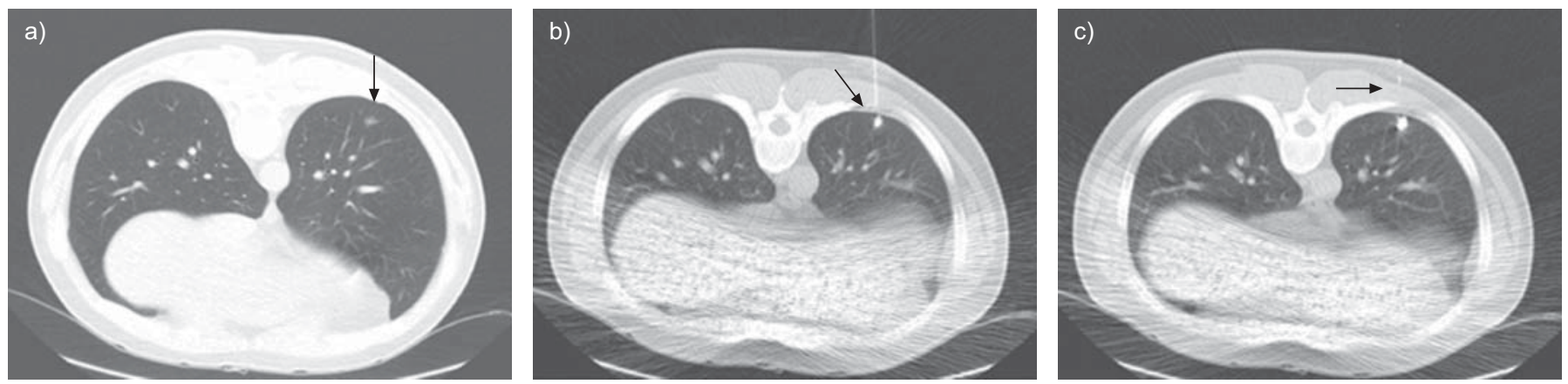

FIGURE 1. A dual localisation with hookwire and lipiodol was performed under intermittent computed tomography fluoroscopy guidance through one introducer needle after the initial single puncture. a) The arrow indicates a ground-glass opacity lesion. b) The arrow indicates lipiodol and the introducer needle. c) The arrow indicates the hookwire.

some negative aspects of the techniques during the operation. The hookwire technique allows precise and quick pre-operative localisation, but has a problem with dislodgement of the hookwire [15-17], air embolism and inaccurate resection margins. Lipiodol is a safe and inexpensive material for localisation [18], but lesions cannot be quickly detected. Thus, we have used a combined technique with the mammographic hookwire and lipiodol for needlescopic biopsy to make up for the weak points in the solitary hookwire and lipiodol techniques.

The aim of this study was to evaluate the usefulness of a dual localisation technique with hookwire and lipiodol performed under CT-fluoroscopy guidance before needlescopy-assisted lung biopsy.

\section{MATERIALS AND METHODS \\ Patients}

All pulmonary nodules that were potentially difficult to localise during surgery were candidates for the pre-operative marking procedure. This procedure was performed when both the radiologists and surgeons thought it might be difficult to find the target lesion by thoracoscopy alone. GGO lesions, small nodules $(<10 \mathrm{~mm})$ or lesions situated under the pulmonary parenchymal surface which could not be detected by needlescopy were included in this study.

Between October 2007 and September 2009, CT-guided marking was performed on 44 pulmonary nodules in 40 patients (23 males and 17 females). Of these, lipiodol marking alone was performed in four patients because they were too close to the diaphragm to localise with a hookwire, and another four patients who were not suitable for the needlescopy-assisted procedure because of pleural adhesions were excluded. Ultimately, 32 patients with 36 nodules were enrolled in the present study.

The patients were divided into two groups according to the objectives of the operation (tissue diagnosis or curative surgery). Operations with curative intent were carried out when small lesions were confirmed as hamartoma by pre-operative percutaneous needle aspiration biopsy, or were strongly suspected to be metastatic lung cancer lesions that could be resected by the thoracoscopic method.

\section{Pre-operative localisation for pulmonary nodule}

Prior to surgery, a dual localisation was performed under intermittent CT (Brilliance 64; Phillips, Amsterdam, the Netherlands) fluoroscopy guidance by one of three board-certified radiologists experienced in CT-guided interventional techniques. After local anaesthesia of the thoracic wall, the introducer needle was advanced into the nodule according to the shortest access route, then $0.2 \mathrm{~mL}$ of lipiodol contained in a 1-mL syringe was injected into the centre of the nodule, and a mammographic hookwire was deployed at the same location (fig. 1). These combined methods were carried out through one introducer needle after the initial single puncture. Immediately after the marking procedure, all patients underwent a CT scan to confirm correct positioning of the lipiodol and the hookwire tip, and to determine whether procedure-related complications, such as pneumothorax or haemorrhage had occurred. Then the patients were transported directly to the operating theatre for surgery.

\section{Operative techniques}

As previously mentioned [3], needlescopy-assisted surgeries were performed under one-lung anaesthesia, using a doublelumen endotracheal tube with three thoracoports (one 11.5- $\mathrm{mm}$ port and two 2-mm miniports). Initially, an incision of $\sim 15 \mathrm{~mm}$ was usually made at the sixth or seventh intercostal space on the anterior axillary line and an $11.5-\mathrm{mm}$ port was inserted through the incision. A 2-mm needlescopy was then introduced into the thoracic cavity through the $11.5-\mathrm{mm}$ port to determine whether any dense adhesions were present and to find the location of the target lesion. If pleural adhesion was observed in the thoracic cavity, the needlescopy-assisted procedure was abandoned. In the remainder, a 2-mm port for 2-mm needlescopy (Hopkins II Forward Oblique-Telescope $0^{\circ}$; Karl-Storz $\mathrm{GmbH}$ and Co, Tuttlingen, Germany) was created at the fifth or sixth intercostal space on the midaxillary line and another 2-mm port was created for a MiniSite Endo Grasp (Covidien, Norwalk, CT, USA) at the fifth or sixth intercostal space on the posterior axillary line. An 11.5-mm port was then used to introduce an endoscopic linear stapler (Endo-GIA 60; Auto Suture Company Division, United States Surgical Corporation, Norwalk or ETH-ECR60D; Ethicon Endo-Surgery, Inc., Cincinnati, OH, USA) or for specimen extraction.

For diagnostic procedures or in cases of palmar hyperhidrosis, not requiring specimen retrieval, 2-mm ports were used exclusively. However, for extirpative needlescopic procedures, requiring pulmonary resection using an endoscopic linear stapler and specimen extraction, one $11.5-\mathrm{mm}$ port was permitted; all remaining ports were of $2-\mathrm{mm}$. No benefit was 
realised by performing the procedure using only needlescopic instrumentation and making an incision for specimen retrieval at the end of the procedure. Therefore, one 11.5- $\mathrm{mm}$ incision was made at the outset of the procedure.

After detecting the hookwire-marked site by needlescopy, the precise site and resection margin of the radiopaque lesion was confirmed under fluoroscopic monitoring in multiple projections and resected with endostaplers (fig. 2). Successful resection of the nodule was finally confirmed by viewing the radiopaque nodule within the resected specimen under $\mathrm{C}$-arm fluoroscopy (fig. 3). The specimen was histologically diagnosed by a routine intraoperative pathological examination.

\section{RESULTS}

Between October 2007 and September 2009, CT-guided dual marking was performed on 36 pulmonary nodules in 32 patients (18 males and 14 females). The mean age of the patients was $58 \pm 12$ (range 12-77) yrs. Table 1 shows the characteristics of the nodules.

The mean time for the dual localisation procedure was $13.1 \pm 4.8$ (7-23) min. Although a small pneumothorax occurred in nine patients and mild intrapulmonary bleeding was observed in three cases, no additional treatment was needed for these complications.

At needlescopy, the hookwire could be easily seen in all cases and was a great help in locating the target lesion. Fluoroscopy showed the radiopaque nodules clearly in all cases. One hookwire dislodged during the operation. Nevertheless, lipiodol markings seen on fluoroscopy guided a successful wedge resection without difficulty. All nodules were successfully resected under needlescopy without conversion to a conventional thoracoscopy (5 $\mathrm{mm}$ or $10 \mathrm{~mm}$ thoracoscopy) or a minithoracotomy.

Table 2 shows the post-operative diagnoses of the localised lesions and details further surgical treatments. 24 nodules were resected for tissue diagnosis and 12 were resected with curative intent. After examining intraoperative frozen sections of the localised lesions, nine patients underwent thoracoscopic lobectomy, and one patient underwent thoracoscopic thymectomy. Subtotal gastrectomy was performed in one patient 7 days after needlescopic wedge resection.

The margins of the resected specimen were adequate and negative in 33 cases. The mean distance from the resected
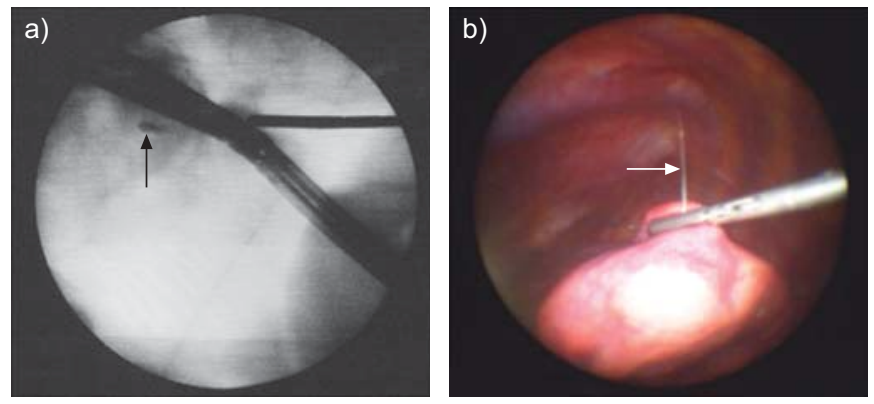

FIGURE 2. a) The lesion which was localised with hookwire and lipiodol was confirmed under fluoroscopy (arrow indicates pulmonary nodule). b) The lesion was quickly detected due to hookwire under needlescope (the arrow indicates hookwire).

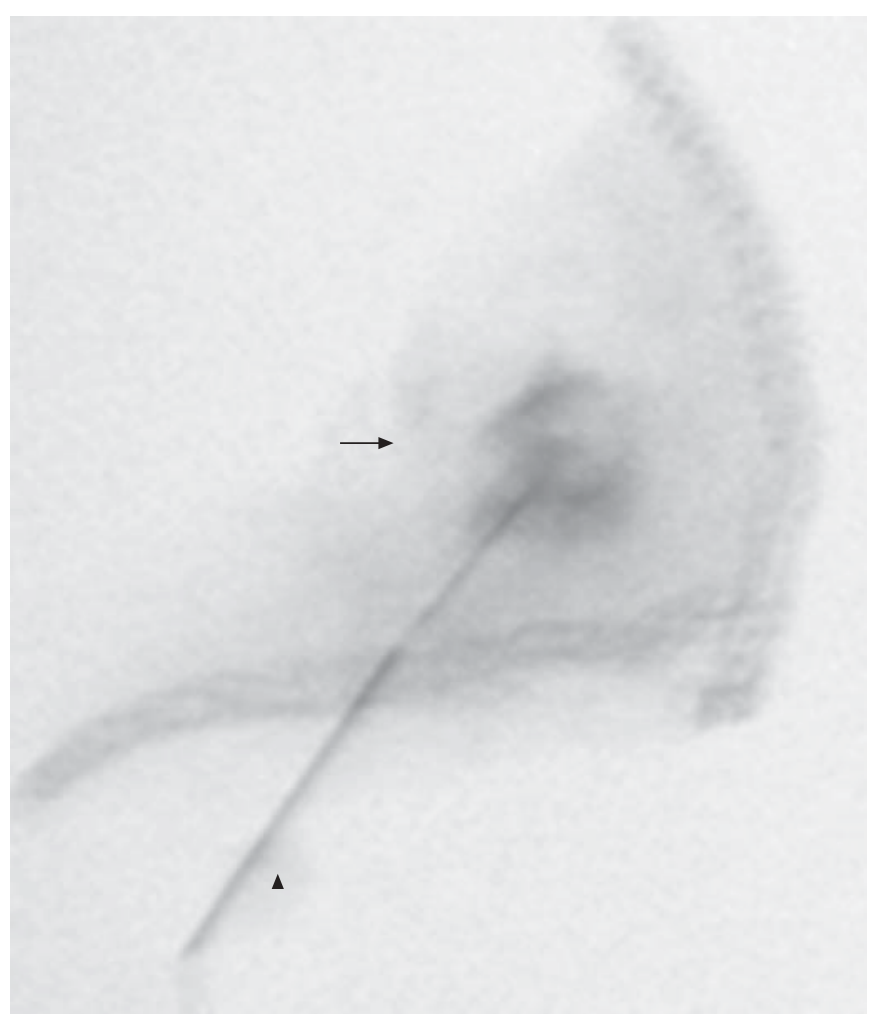

FIGURE 3. The resected specimen that had adequate resection margins is shown. The arrow indicates lipiodol injected at the pulmonary nodule, the arrowhead indicates hookwire.

margin to the lesion was $4.9 \pm 4.5(0-15) \mathrm{mm}$. Additional wedge resection was needed in three cases and negative resection margins were achieved in all three cases.

There was no complication related to needlescopy-assisted resection. With the exception of three patients who required an additional wedge resection and 10 patients who underwent a further procedure (one thymectomy and nine lobectomies), the mean operation time was $34.4 \pm 14.7(18-75) \mathrm{min}$, and the mean duration of the indwelling chest tube was $2.2 \pm 0.73$ (1-4) days.

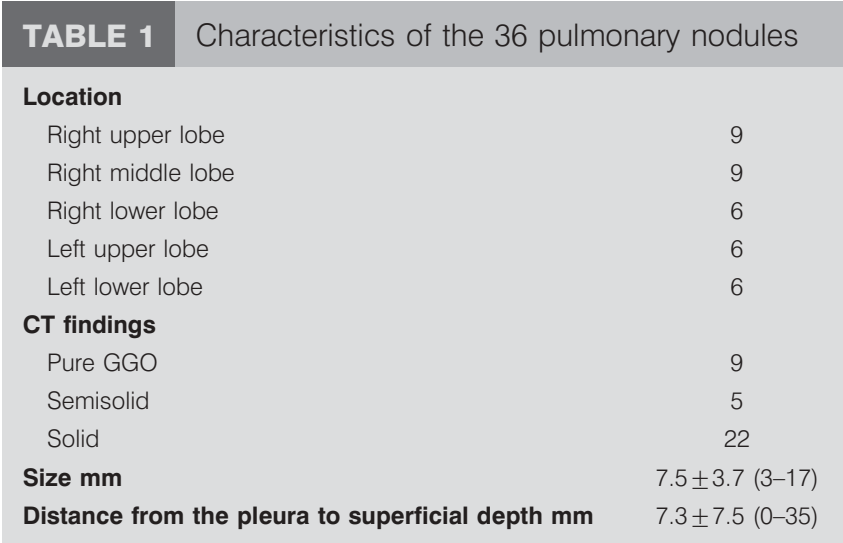

Data are presented as $\mathrm{n}$ or mean $\pm \mathrm{SD}$ (range). CT: computer tomography GGO: ground-glass opacity. 


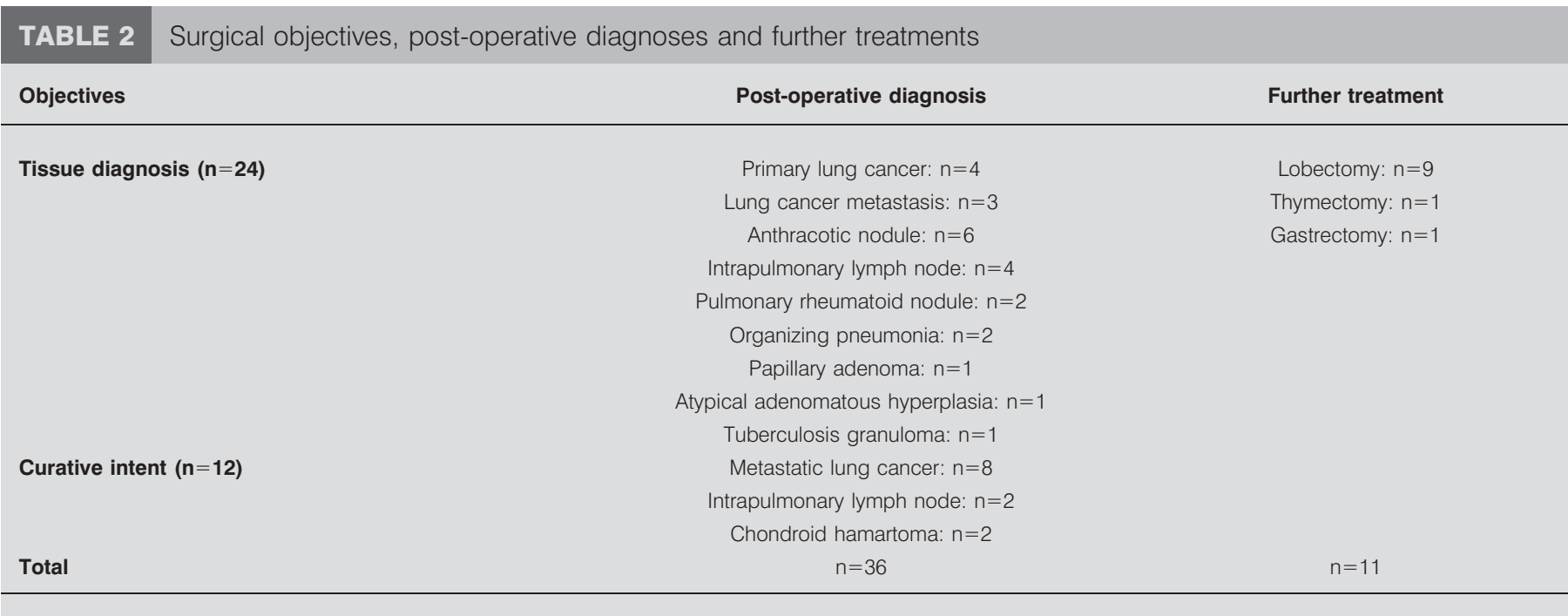

\section{DISCUSSION}

Needlescopic procedure was defined as surgery using needlescopic instruments that have a diameter of $<3 \mathrm{~mm}$ [19]. In thoracic procedures, this surgical technique had initially been applied mainly to diagnostic thoracoscopy [20], thoracic sympathectomy for palmar hyperhidrosis [21] and laser ablation for spontaneous pneumothorax [22]. Recently, lung biopsies for interstitial lung disease and pulmonary resections for indeterminate pulmonary nodules have been introduced [3, 23]. Needlescopic lung surgery is much less invasive compared with more conventional procedures and is technically feasible and safe [3]. Furthermore, needlescopic lung resection seems to result in less post-operative pain and scarring than conventional thoracoscopy. Accordingly, the appropriate use of 2-mm instrumentation is likely to enhance patient satisfaction by minimising incisionrelated morbidities and improving cosmesis.

However, it is very difficult to grasp the lung firmly with miniforceps because of the short jaws and poor holding strength, and the lung is easily injured when the lesion had been grasped. For these reasons, a localisation method which was different from conventional thoracoscopic methods was needed.

Conventional thoracoscopic resection of small peripheral pulmonary nodules by digital palpation is both straightforward and rapid when a nodule is in contact with the pleural surface. On the contrary, when a nodule is too small or deep to the pleural surface, digital palpation may not be possible and this may result in conversion to open surgery. This appears to be more frequent if the distance between the nodule and the nearest pleural surface is $>5 \mathrm{~mm}$ and when the nodule is $\leqslant 10 \mathrm{~mm}$ in size [24]. Furthermore, because GGO lesions cannot usually be palpated or visualised during surgery regardless of lesion size or depth, localisation by digital palpation is not possible and, thus, several marking techniques have been developed for the pre-operative and intraoperative localisations of these lesions.

In our institution, the hookwire method was initially used in needlescopy-assisted surgeries in order to detect the lesions quickly and reduce lung injury by using the hookwire, instead of the lung surface, as the grasping portion.
However, excessive traction on the grasped hookwire to ensure the resection margin caused hookwire dislodgement, making it very difficult to detect and resect pulmonary lesions. In addition, even though the resection margin was negative, it is still difficult to maintain a proper resection margin $(\geqslant 10 \mathrm{~mm}$ or double lengths of tumour itself) with the single hookwire method in the case of malignant diseases.

For these reasons, a dual localisation method with hookwire and lipiodol was thought to be needed in needlescopy-assisted pulmonary resection.

The marking procedure with lipiodol has the following advantages: 1) overresection of the normal lung tissue around the nodules is prevented because lipiodol marks the nodules as clear spots that are $<1 \mathrm{~cm}$ in size during fluoroscopy; 2) the lipiodol remains up to 3 months after the marking, which solves the problem of requiring both a CT and the operating room simultaneously; 3) although the barium marking procedure affects pathological findings caused by the inflammatory response and barium itself, lipiodol did not affect the pathological findings; and 4) even in the case of deeply situated nodules, the lipiodol marking could easily localise the nodules as a clear spot because it diffused only to a small extent [18]. Because of these advantages, lipiodol was chosen as the dual marking material.

Although the dual localisation method was used in needlescopy-assisted surgery, the margins of the resected specimen were positive in three cases in this study. This result was thought to be caused by an old model of C-arm fluoroscopic equipment which was not good at identifying the lipiodol marking accurately. After the old model of C-arm fluoroscopy was changed for a new one, all margins of the resected specimen were adequate and negative in 13 cases. Furthermore, after first resection, because the stapled site of the lung was used as the grasp site, we could perform an additional wedge resection safely without lung injury and achieve a negative resection margin in all three cases.

The mean time for the dual localisation procedure was $13.1 \pm 4.8$ (7-23) $\mathrm{min}$ and minimal complications were observed in 12 
(33.3\%) cases. Before the dual marking procedure, the hookwire method was performed in eight cases in our institution. The mean time for the hookwire localisation was $9.1 \pm 3.8 \mathrm{~min}$ and two $(25 \%)$ complications (one pneumothorax and one haemothorax) occurred. Although the mean time for the dual marking procedure was thought to be longer than the hookwire, statistically, there was no significant difference in localisation time $(\mathrm{p}=0.06)$ and complication rate $(\mathrm{p}=0.50)$ between the two localisation methods.

There were further procedures performed in 11 cases (nine lobectomies, one gastrectomy and one thymectomy) according to the intraoperative frozen section reports after needlescopyassisted lung resection. The rest of these cases did not need further surgical treatment because the pulmonary lesions were confirmed as benign or properly resected for treatment, and these patients could quickly recover and be discharged. We thought that needlescopy-assisted lung resection for undetermined nodules or GGO lesions under the dual marking procedure was effective in obtaining a histological diagnosis compared with conventional thoracoscopy. To the best of our knowledge, no previous reports have described this combined method, especially under needlescopy-assisted lung resection.

In conclusion, the combined method of mammographic hookwire anchoring and lipiodol injection under CT fluoroscopy is safe and not time-consuming, and needlescopyassisted lung resection for small nodules or GGO lesions is effective for histological diagnosis and treatment in some cases. Importantly, this technique has now made it possible both to detect small pulmonary lesions quickly without lung injury and to evaluate whether the lines of resection will achieve the required margins for pulmonary lesions under needlescopic lung resection.

\section{STATEMENT OF INTEREST}

None declared.

\section{REFERENCES}

1 Yeow KM, Tsay PK, Cheung YC, et al. Factors affecting diagnostic accuracy of CT-guided coaxial cutting needle lung biopsy: retrospective analysis of 631 procedures. J Vasc Interv Radiol 2003; 14: 581-588.

2 Ikeda Y, Miyoshi S, Seki N, et al. Needlescopic operation for partial lung resection. Ann Thorac Surg 2003; 75: 599-601.

3 Kim HK, Jo W-M, Jung JH, et al. Needlescopic lung biopsy for interstitial lung disease and indeterminate pulmonary nodules: a report on 65 cases. Ann Thorac Surg 2008; 86: 1098-1103.

4 Dendo S, Kanazawa S, Ando A, et al. Preoperative localization of small pulmonary lesions with a short hook wire and suture system: experience with 168 procedures. Radiology 2002; 225: 511-518.

5 Wicky S, Dusmet M, Doenz F, et al. Computed tomographyguided localization of small lung nodules before video-assisted resection: experience with an efficient hook-wire system. $J$ Thorac Cardiovasc Surg 2002; 124: 401-403.

6 Sortini D, Feo CV, Carcoforo P, et al. Thoracoscopic localization techniques for patients with solitary pulmonary nodule and history of malignancy. Ann Thorac Surg 2005; 79: 258-262.
7 Shennib H. Intraoperative localization techniques for pulmonary nodules. Ann Thorac Surg 1993; 56: 745-748.

8 Nomori H, Horio $\mathrm{H}$, Naruke $\mathrm{T}$, et al. Fluoroscopy-assisted thoracoscopic resection of lung nodules marked with lipiodol. Ann Thorac Surg 2002; 74: 170-173.

9 Mayo JR, Clifton JC, Powell TI, et al. Lung nodules: CT-guided placement of microcoils to direct video-assisted thoracoscopic surgical resection. Radiology 2009; 250: 576-585.

10 Magistrelli P, D'Ambra L, Berti S, et al. Use of India ink during preoperative computed tomography localization of small peripheral undiagnosed pulmonary nodules for thoracoscopic resection. World J Surg 2009; 33: 1421-1424.

11 Poretti FP, Brunner E, Vorwerk D. Einfache Lokalisation von intrapulmonalen Rundherden - CT-gesteuerte perkutane Hakenmarkierung. [Simple localization of peripheral pulmonary nodules - CT-guided percutaneous hook-wire localization]. Rofo 2002; 174: 202-207.

12 McConnell PI, Feola GP, Meyers RL. Methylene blue-stained autologous blood for needle localization and thoracoscopic resection of deep pulmonary nodules. J Pediatr Surg 2002; 37: 1729-1731.

13 Jimenez MF. Prospective study on video-assisted thoracoscopic surgery in the resection of pulmonary nodules: 209 cases from the Spanish Video-Assisted Thoracic Surgery Study Group. Eur J Cardiothorac Surg 2001; 19: 562-565.

14 Kondo R, Yoshida K, Hamanaka K, et al. Intraoperative ultrasonographic localization of pulmonary ground-glass opacities. J Thorac Cardiovasc Surg 2009; 138: 837-842.

15 Gonfiotti A, Davini F, Vaggelli L, et al. Thoracoscopic localization techniques for patients with solitary pulmonary nodule: hookwire versus radio-guided surgery. Eur J Cardiothorac Surg 2007; 32: 843-847.

16 Ciriaco P, Negri G, Puglisi A, et al. Video-assisted thoracoscopic surgery for pulmonary nodules: rationale for preoperative computed tomography-guided hookwire localization. Eur J Cardiothorac Surg 2004; 25: 429-433.

17 Thaete FL, Peterson MS, Plunkett MB, et al. Computed tomography-guided wire localization of pulmonary lesions before thoracoscopic resection: results in 101 cases. J Thorac Imaging 1999; 14: 90-98.

18 Watanabe $\mathrm{K}$, Nomori $\mathrm{H}$, Ohtsuka $\mathrm{T}$, et al. Usefulness and complications of computed tomography-guided lipiodol marking for fluoroscopy-assisted thoracoscopic resection of small pulmonary nodules: experience with 174 nodules. J Thorac Cardiovasc Surg 2006; 132: 320-324.

19 Gagner M, Garcia-Ruiz A. Technical aspects of minimally invasive abdominal surgery performed with needlescopic instruments. Surg Laparosc Endosc 1998; 8: 171-179.

20 d'Alessandro AA. Microthoracoscopy: at the cutting edge of thoracic surgery. J Laparoendosc Adv Surg Tech A 1997; 7: 313-318.

21 Yamamoto H, Kanehira A, Kawamura M, et al. Needlescopic surgery for palmar hyperhidrosis. J Thorac Cardiovasc Surg 2000, 120: $276-279$.

22 Hazama K, Akashi A, Shigemura N, et al. Less invasive needle thoracoscopic laser ablation of small bullae for primary spontaneous pneumothorax. Eur J Cardiothorac Surg 2003; 24: 139-144.

23 Yamada S, Kohno T, Abe Y. Video-assisted S6b-subsegmental resection after computed tomography guided localization of pulmonary nodules. Jpn J Thorac Cardiovasc Surg 2003; 51: 626-629.

24 Suzuki K, Nagai K, Yoshida, J, et al. Video-assisted thoracoscopic surgery for small indeterminate pulmonary nodules - Indications for preoperative marking. Chest 1999; 115: 563-568. 